\title{
Quest for localized 4D black holes in brane worlds. II. Removing the bulk singularities
}

\author{
P. Kanti \\ CERN, Theory Division, CH-1211 Geneva 23, Switzerland \\ I. Olasagasti \\ Physics Department, University of Ioannina, GR-451 10 Ioannina, Greece \\ K. Tamvakis \\ CERN, Theory Division, CH-1211 Geneva 23, Switzerland \\ and Physics Department, University of Ioannina, GR-451 10 Ioannina, Greece
}

(Received 27 July 2003; published 1 December 2003)

\begin{abstract}
We analyze further the possibility of obtaining localized black hole solutions in the framework of RandallSundrum-type brane-world models. We consider black hole line elements analytic at the horizon, namely, generalizations of the Painlevé and Vaidya metrics, which are taken to have a decaying dependence of the horizon on the extra dimension. These backgrounds have no other singularities apart from the standard black hole singularity which is localized in the direction of the fifth dimension. Both line elements can be sustained by a regular, shell-like distribution of bulk matter of a non-standard form. Of the two, the Vaidya line element is shown to provide the most attractive, natural choice: despite the scaling of the horizon, the five-dimensional spacetime has the same topological structure as the one of a Randall-Sundrum-Schwarzschild spacetime and demands a minimal bulk energy-momentum tensor.
\end{abstract}

DOI: $10.1103 /$ PhysRevD.68.124001

PACS number(s): 04.50.+h, 04.70.Bw, 11.10.Kk, 11.25.Mj

\section{INTRODUCTION}

Higher-dimensional models of gravitation have attracted considerable interest in the last few years, mostly motivated by the need to explain the large difference in magnitude between the Planck scale $M_{P} \sim 2 \times 10^{18} \mathrm{GeV}$ and the electroweak scale of particle physics. A number of interesting theoretical ideas have arisen from this framework [1-3]. Of particular interest is the idea of an extra non-compact dimension [3] realized in the models proposed by Randall and Sundrum (RS), where the spacetime corresponds to regions of AdS space separated by zero-thickness 3-branes. The standard model interactions are confined on such a 3-brane, while gravitation, propagating in the five-dimensional (5D) bulk, is represented on the brane by an ordinary massless graviton dynamically localized on it [4]. The fourdimensional (4D) Planck mass is an effective scale given in terms of the fundamental scale of five-dimensional gravity and the AdS radius of the five-dimensional spacetime. Apart from the study of their cosmological implications, the above higher-dimensional models have triggered intense research activity on the topic of black holes in the context of scenarios with both warped [5-7] and large extra dimensions [8].

In the standard four-dimensional world, gravitational collapse is described by the four-dimensional Schwarzschild metric. In a five-dimensional framework, it would be natural to expect that the $(3+1)$-dimensional spacetime could still be described by a Schwarzschild-like metric when matter localized on the brane undergoes gravitational collapse. For instance, the one-brane RS model accepts a RS-Schwarzschild black hole solution with a factorized metric $g_{M N}$ $=\operatorname{Diag}\left(e^{-2 \lambda|y|} g_{\mu \nu}^{(S)}, 1\right)$, with $g_{\mu \nu}^{(S)}$ the standard fourdimensional Schwarzschild metric. This solution corresponds to a black string, infinite in the fifth dimension [9], that re- duces to a usual Schwarzschild metric on the brane. Although the five-dimensional Ricci scalar and the square of the Ricci tensor corresponding to this solution are everywhere finite, the square of the Riemann tensor diverges at the AdS horizon as $e^{4 \lambda|y|} / r^{6}$. This singularity renders the above solution physically unsuitable and has led to the speculation that there exist localized black cigar solutions of a finite extension along the fifth dimension arising from a GregoryLaflamme type of instability [10] near the AdS horizon.

The possibility of obtaining localized black hole solutions in brane models was investigated in a recent article by two of the present authors [11]. There, in the framework of a fivedimensional theory with a warped metric of the RandallSundrum type $g_{M N}=\operatorname{Diag}\left(e^{2 A(y)} g_{\mu \nu}, 1\right)$, a dependence of $g_{\mu \nu}$ on the extra dimension was introduced. A particular ansatz corresponding to a 4D Schwarzschild black hole with a "decaying" horizon scaled as $r_{h}(y)=r_{h} e^{-a y^{2}}$ was employed. The bulk energy-momentum tensor sustaining such a behavior was derived and was shown not to correspond to that arising from a conventional form of bulk matter (scalar or gauge field). For such an exotic, shell-like matter distribution in the bulk, the black hole singularity is indeed localized near the brane and the spacetime is well defined near the AdS horizon, in contrast to the behavior found in blackstring-type solutions.

Nevertheless, the above behavior was plagued by the appearance of a second, although localized, singularity at the black hole horizon due to the singular behavior of the bulk energy-momentum tensor at this point. An alternative choice for the five-dimensional metric, which describes a spacetime with a distinctly different topology from the first one but which also reduces to the same black hole solution on the brane, was shown to be free of this singularity. Unfortunately, this choice failed to avoid the singularity at the AdS 
horizon. Thus, it was speculated but not demonstrated that the additional bulk singularity located at the black hole horizon is not a generic feature of every five-dimensional spacetime that induces a black hole solution on the brane, but that an alternative, well-defined 5D non-factorized spacetime indeed exists, sustained by an equally well-defined distribution of bulk matter, possibly of a non-standard form.

In the present paper, we pursue further the above open question. We first demonstrate that the non-factorized embedding of a non-analytic 4D black hole line element (i.e. with a diverging, at the horizon, $g_{r r}$ metric component), in a 5D spacetime, results into the manifestation of the black hole horizon as a true spacetime singularity in the fivedimensional curvature invariant quantities. We then proceed to introduce alternative metric ansätze that describe the same four-dimensional background but which are analytic at the black hole horizon, namely, the Painlevé and Vaidya spacetimes. A dependence on the fifth dimension of a decaying form is introduced into the horizon as in the previous study of the problem [11]. Both ansätze lead to five-dimensional backgrounds with no other singularity apart from the standard black hole singularity at $r=0$, which, in addition, turns out to be localized near the brane along the fifth dimension. These backgrounds are promoted into solutions of Einstein's equations of motion if we introduce a bulk energymomentum tensor resulting from a shell-like matter distribution localized near the brane and not attributable to standard forms of bulk matter (scalar, gauge fields, etc.). The Painlevé ansatz demands the introduction of a complicated bulk energy-momentum tensor, which is well defined at the black hole horizon. On the other hand, the Vaidya ansatz leads to a minimal model: despite the non-trivial profile of the black hole horizon along the extra coordinate, all curvature invariants for this background have the form of the ones of a factorized RS-Schwarzschild spacetime; the simplicity of the resulting background is then shown to demand the introduction of only two non-trivial pressure components in the bulk, a feature often encountered in brane-world models.

\section{NON-FACTORIZED RS-SCHWARZSCHILD SPACETIMES}

In what follows we shall review, in a relatively selfcontained way, the results obtained in the framework of a non-factorized Randall-Sundrum-Schwarzschild spacetime, an ansatz introduced in Ref. [11]. In addition, we shall consider generalizations of the previously studied ansätze and examine their singularity properties. The results of this analysis will pave the way for the study of black hole metrics which are analytic at the horizon and describe localized black hole configurations.

Looking for black hole solutions that are localized around the brane, we introduce for the metric a non-factorizable ansatz of the form

$$
d s^{2}=g_{M N} d x^{M} d x^{N}=e^{2 A(y)} \hat{g}_{\mu \nu}(x, y) d x^{\mu} d x^{\nu}+d y^{2} .
$$

Note that a $y$-dependent coefficient of $d y^{2}$ has been set to unity through a Weyl rescaling. The $\hat{g}_{\mu \nu}(x, y)$ metric tensor is assumed to reduce to the usual Schwarzschild one at the location of the brane at $y=0$, i.e. $\hat{g}_{\mu \nu}(x, 0)=g_{\mu \nu}^{(S)}(x)$, while the $y$ dependence will provide an additional scaling of the value of the black hole horizon with respect to the extra dimension, independently of the one of the warp factor. If this $y$ dependence is appropriately chosen, the horizon may decay at increasing distance from the brane and eventually vanish well before the AdS horizon, thus leading to the desirable localization of the black hole along the extra dimension and the avoidance of the singularity at the AdS horizon.

Our general framework will be the five-dimensional gravitational theory described by the action

$$
S=-\int d^{4} x d y \sqrt{-g}\left\{-\frac{R}{2 \kappa_{5}^{2}}+\Lambda_{B}+\frac{\sigma}{\sqrt{g_{55}}} \delta(y)-\mathcal{L}_{B}\right\},
$$

where $\kappa_{5}^{2}=8 \pi G_{5}$, with $G_{5}$ the five-dimensional Newton's constant, and $\Lambda_{B}$ stands for a bulk cosmological constant. In addition, $\mathcal{L}_{B}$ represents any additional existing bulk matter, and the constant $\sigma$ denotes the positive tension of the brane located, as noted above, at $y=0$.

In Ref. [11] we concentrated on the case of a spherically symmetric line element on the brane, and chose the ansatz

$$
\begin{aligned}
d s^{2}= & e^{2 A(y)}\left\{-U(r, y) d t^{2}+U^{-1}(r, y) d r^{2}\right. \\
& \left.+r^{2}\left(d \theta^{2}+\sin ^{2} \theta d \varphi^{2}\right)\right\}+d y^{2} .
\end{aligned}
$$

We also assumed that the five-dimensional spacetime is $\mathcal{Z}_{2}$ symmetric and therefore invariant under the mirror transformation $y \rightarrow-y$. For $U=U(r)$ and a vanishing cosmological constant $\Lambda$ on the brane, the above metric ansatz reduces to the black string solution [9] with $A(y)=-\lambda|y|$, where $\lambda^{2}$ $=\kappa_{5}^{2}\left|\Lambda_{B}\right| / 6$, and $U(r)=1-(2 M / r)$. For $\Lambda \neq 0$, this solution can be easily generalized to the RS-AdS/dSSchwarzschild one [12]. Both types of five-dimensional solutions have finite $R$ and $R_{M N} R^{M N}$, while the square of the Riemann tensor is given by

$$
R_{M N R S} R^{M N R S} \propto \frac{48 M^{2}}{r^{6}} e^{-4 A(y)} .
$$

The above reveals the existence of a string-like black hole singularity infinitely extending along the extra dimension as well as an additional singularity at the point where the warp factor vanishes, either at the AdS horizon for a flat brane, or at a finite coordinate distance for a de Sitter brane.

The introduction of $y$ dependence in the metric function $U$ and, therefore, the resulting scaling of the horizon or, equivalently, the "mass" of the black hole, with the distance from the brane, could provide a resolution to both of the above problems. If $M(y)$ decreases faster than the function $e^{-2 A(y)}$ increases, then the singular term in Eq. (4) vanishes before the spatial limit of the extra dimension is reached. Unfortunately, as it was shown in [11], an empty bulk or a bulk with a single scalar or gauge field cannot support such a $y$ dependence. We then followed an alternative approach by demanding an exponentially decaying black hole horizon, i.e. 
$U(r, y)=1-w(y) / r$, with $w(y)=2 M e^{-a y^{2}}$ and determining the components of the unknown bulk energy-momentum tensor through Einstein's equations. This particular ansatz was found to demand the introduction of three independent nontrivial components of the bulk energy-momentum tensor, namely $T_{t}^{t}, T_{r}^{r}$ and $T_{r 5}$. All three were shown to correspond to a localized shell-like distribution of exotic matter around the brane and to vanish at both $r$ and $y$ infinity.

Although the singular term in Eq. (4) decayed exponentially with the distance from the brane, thus, localizing the black hole singularity and removing the one from the boundary of the extra dimension, a new problem arose. The harmless, in 4 dimensions, horizon non-analyticity at $r=w(y)$ was transformed into a true singularity of the fivedimensional spacetime. All components of the bulk energymomentum tensor diverged at the location of the horizon with the same singularity arising in all curvature invariant quantities through the appearance of additional terms singular at $r=w(y)$. For instance, the scalar curvature took the form $R=-20 A^{\prime 2}-8 A^{\prime \prime}-w^{\prime 2} / 2(r-w)^{2}$. Although all singular terms appearing in the invariant quantities also decay exponentially away from the brane, the question of whether the appearance of a second singularity is unavoidable in trying to localize a bulk string-like black hole singularity remained open.

We will now show that the embedding in a higherdimensional spacetime of a brane line element that is characterized by the presence of a horizon and of mixed coordinate dependence, i.e. dependence on both brane and bulk coordinates, quite generally results in the manifestation of brane horizons as true, bulk singularities of the higherdimensional theory. This result turns out to be independent of the dimensionality of the problem and can be demonstrated for a more general four-dimensional metric ansatz than the one initially chosen. We may therefore consider the following Schwarzschild-like line element:

$$
d \hat{s}_{p}^{2}=-U^{2}(r, y) d t^{2}+\frac{d r^{2}}{V^{2}(r, y)}+r^{2} d \Omega_{p-2}^{2},
$$

describing a $(p+1)$-dimensional brane, embedded in a $D$-dimensional spacetime of the form

$$
\begin{aligned}
d s^{2} & =g_{M N} d x^{M} d x^{N} \\
& =e^{2 A(y)} \hat{g}_{\mu \nu}(x, y) d x^{\mu} d x^{\nu}+\gamma_{m n}(y) d y^{m} d y^{n} .
\end{aligned}
$$

The existence of true spacetime singularities will be reflected in the expressions of the $D$-dimensional curvature invariant quantities. For our purposes, the expression of the scalar curvature will be adequate. In the Appendix, we give the nonvanishing Christoffel symbols and the components of the Ricci tensor that lead to the evaluation of this quantity.

In the restricted case where $V=U$, the determinant $\sqrt{-\hat{g}}$ is $y$ independent, and, therefore, all terms in the expression of the scalar curvature in the Appendix involving derivatives of $\ln \sqrt{-\hat{g}}$ with respect to the extra coordinates will vanish. Thus, the singular terms in the expression of $R$ are

$$
\frac{1}{4} \hat{g}^{\mu \nu} \hat{g}^{\rho \lambda} \hat{g}_{\nu \lambda, m} \hat{g}_{\mu \rho}^{, m}=-\frac{2}{U^{2}} \gamma^{m n} \partial_{m} U \partial_{n} U
$$

Since the $(p+1)$-dimensional metric describes a Schwarzschild-like black hole on the brane, $U(r, y)$ must vanish at the horizon. Then, the above quantity diverges transforming the $(p+1)$-dimensional coordinate singularity to a true singularity of the $D$-dimensional spacetime. A special case of the above is the ansatz (3) used in [11] where the presence of a horizon on the 3-brane led indeed to the appearance of a second bulk singularity.

Let us go back to the general case of different $U$ and $V$ metric functions and let us put together all terms from Eq. (A4) involving solely derivatives of the metric tensor $\hat{g}_{\mu \nu}$ and its determinant, which is now obviously $y$ dependent. Then, we end up with

$$
\begin{aligned}
& -2 \widetilde{D}^{m} \widetilde{D}_{m} \ln \sqrt{-\hat{g}}-\partial^{m} \ln \sqrt{-\hat{g}} \partial_{m} \ln \sqrt{-\hat{g}} \\
& +\frac{1}{4} \hat{g}^{\mu \nu} \hat{g}^{\rho \lambda} \hat{g}_{\nu \lambda, m} \hat{g}_{\mu \rho}{ }^{m}+\frac{1}{2} \hat{g}_{\mu \nu, m} \hat{g}^{\mu \nu, m} \\
& =-2 \gamma^{m n}\left(\frac{\partial_{m} \partial_{n} U}{U}-\frac{\partial_{m} \partial_{n} V}{V}+2 \frac{\partial_{m} V}{V} \frac{\partial_{n} V}{V}\right. \\
& \left.-\frac{\partial_{m} U}{U} \frac{\partial_{n} V}{V}\right) .
\end{aligned}
$$

Another term that might also diverge at the location of the horizon is proportional to

$$
\partial^{m} A \partial_{m} \ln \sqrt{-\hat{g}}=\partial^{m} A\left(\frac{\partial_{m} U}{U}-\frac{\partial_{m} V}{V}\right) .
$$

If we want the metric functions to be chosen independently of the warp factor function $A(y)$, then the singular combination inside brackets in the last expression must vanish. In the case where only $U$ vanishes, or $U$ and $V$ vanish at different values of $r$, the singularity is unavoidable. In the case where both $U$ and $V$ vanish at the same value of $r$, the only solution that leads to the vanishing of this combination term is $U(r, y)=g(r) V(r, y)$. But then, Eq. (8) is always plagued by a singularity. If, on the other hand, we allow for the metric functions and the warp factor to be related, then, it is the combination of Eqs. (8) and (9) that must vanish instead. In principle, one cannot exclude the possibility that a metric ansatz, that could render the scalar curvature finite, may be found. Nevertheless one has to ensure that all curvature quantities become finite at the same time. Despite our attempts, we have not been able to find such a metric ansatz.

Clearly, the key to the removal of the additional bulk singularities is the use of a brane line element that describes a black hole solution but that is analytic at the horizon. In four dimensions, one can use different line elements related by coordinate transformations in order to describe the same spacetime. However, the embedding of these line elements in an extra spacetime automatically breaks their equivalence. The resulting higher-dimensional line elements no longer describe the same spacetime, a fact which is reflected in the 
different sets of curvature invariant quantities determined for each line element. In [11], a Kruskal-Szekeres brane line element, that also describes a black hole solution but without possessing a horizon, was used instead of the Schwarzschild one. It was shown that the corresponding five-dimensional spacetime was free of the presence of the additional singularity. Nevertheless, this particular choice failed to either eliminate the singularity at the AdS horizon or to localize the black hole singularity. In the next two sections, we will study two alternative, analytic, five-dimensional line elements, with different topological structures but both describing a black hole on the brane that will be shown to achieve all of the above goals.

\section{THE NON-FACTORIZED RS-PAINLEVÉ SPACETIME}

Let us start by considering a five-dimensional nonfactorized metric ansatz that contains a Painlevé-type brane line element [13]. The four-dimensional Painlevé line element has been more recently employed in the study of black hole quantum mechanics [14]. A non-factorized 5D version of it can be written as

$$
\begin{aligned}
d s^{2}= & e^{2 A(y)}\left[-\left(1-\frac{2 m(y)}{r}\right) d v^{2}-2 \sqrt{\frac{2 m(y)}{r}} d v d r+d r^{2}\right. \\
& \left.+r^{2}\left(d \theta^{2}+\sin ^{2} \theta d \varphi^{2}\right)\right]+d y^{2}
\end{aligned}
$$

At the location of the brane, at $y=0$, we demand the mass function to reduce to the usual ADM mass parameter of the black hole, i.e. $m(0)=M$. Then, the induced metric on the brane takes the form of the Schwarzschild solution under the coordinate transformation

$$
v \rightarrow t=v+4 M\left(\sqrt{\frac{r}{2 M}}-\operatorname{arctanh} \sqrt{\frac{r}{2 M}}\right) .
$$

The advantage of the line element (10) compared to the one given in Eq. (3) is the fact that, although both describe the same spacetime, the former is analytic at the horizon, i.e. no divergences appear in the metric components at $r=m(y)$.

Hopefully, that will lead to the avoidance of the additional bulk singularity that plagued the Schwarzschild choice. On the other hand, the $y$-dependence of the mass function, or equivalently of the horizon, will be used in the same way in order to localize the black-hole singularity close to the brane and resolve the singularity at the AdS horizon.

From the five-dimensional perspective, the metric ansatz (10) must satisfy Einstein's equations that are found to have the form

$$
\begin{aligned}
6 A^{\prime 2} & +3 A^{\prime \prime}+\frac{2 A^{\prime} m^{\prime}}{r}+\frac{m^{\prime \prime}}{2 r}-\frac{m^{\prime 2}}{8 r m} \\
= & \kappa_{5}^{2}\left[-\Lambda_{B}+S_{v}^{v} \delta(y)+\hat{T}_{v}^{v}\right]
\end{aligned}
$$

$$
\begin{gathered}
6 A^{\prime 2}+3 A^{\prime \prime}-\frac{2 A^{\prime} m^{\prime}}{r}-\frac{m^{\prime \prime}}{2 r}-\frac{m^{\prime 2}}{8 r m} \\
=\kappa_{5}^{2}\left[-\Lambda_{B}+S_{r}^{r} \delta(y)+\hat{T}_{r}^{r}\right] \\
6 A^{\prime 2}+3 A^{\prime \prime}-\frac{m^{\prime 2}}{8 r m}=\kappa_{5}^{2}\left[-\Lambda_{B}+S_{\theta}^{\theta} \delta(y)+\hat{T}_{\theta}^{\theta}\right] \\
\left.\sqrt{\frac{2 m}{r}\left(\frac{A^{\prime} m^{\prime}}{m^{\prime 2}}+\frac{m^{\prime 2}}{8 r m}=\kappa_{5}^{2}\left(-\Lambda_{B}+\hat{T}_{y}^{y}\right)\right.}-\frac{m^{\prime 2}}{8 m^{2}}\right)=\kappa_{5}^{2}\left[S_{r}^{v} \delta(y)+\hat{T}_{r}^{v}\right] \\
-\frac{m^{\prime}}{4 r^{2}} \sqrt{\frac{2 m}{r}}\left(1+\frac{3 r}{2 m}\right)=\kappa_{5}^{2} \hat{T}_{v y} \\
\frac{m^{\prime}}{4 r^{2}}=\kappa_{5}^{2} \hat{T}_{r y},
\end{gathered}
$$

where $S_{\mu \nu}$ is the energy-momentum tensor localized on the brane, the simplest case being that of a brane with a constant tension, namely, $S_{\mu}^{\nu}=\sigma h_{\mu}^{\nu}$. We will see below that for a general mass function $m(y)$ this may be non-trivial, that is, the brane may not be a vacuum wall. In the above, we have also assumed that the total energy-momentum tensor in the bulk, $T_{M N}$, can be written as the sum of two contributions, one coming from the bulk cosmological constant and one from the presence of a bulk matter distribution related to $\mathcal{L}_{B}$, that is

$$
T_{M N}=-g_{M N} \Lambda_{B}+\hat{T}_{M N}
$$

For the remainder of this paper, we will be assuming that a negative bulk cosmological constant gives rise to the usual Randall-Sundrum form of the warp factor $e^{A(y)}=e^{-\lambda|y|}$. Then, the terms on the left-hand side (lhs) of the diagonal components of Einstein's equations that are proportional to $A^{\prime 2}$ and $A^{\prime \prime}$ are cancelled by the presence of $\Lambda_{B}$ on the right-hand side (rhs). All remaining terms containing derivatives of the metric function $m(y)$ are attributed to the presence of the bulk energy-momentum tensor $\hat{T}_{M N}$. In other words, the non-trivial profile of the horizon of the black hole along the extra dimension demands the introduction of a bulk matter distribution. For this particular metric ansatz, it is necessary to introduce non-trivial bulk energy density and diagonal pressure components as well as three off-diagonal ones. Since we are reading the energy-momentum tensor from the Einstein equations, the conservation equation $D_{M} T_{N}^{M}=0$ is trivially satisfied for an arbitrary function $m(y)$.

Having introduced a brane line element with no apparent horizon, we expect that no additional singularity, apart from the black-hole one, will plague the five-dimensional spacetime. By looking at the components of the energymomentum tensor, we can immediately see that they do not 
diverge at any other point apart from the one at $r=0$. Nevertheless, we should check the expressions of the curvature invariant quantities which are directly related to the singularity structure of spacetime. The scalar curvature is

$$
R=-20 \lambda^{2}+\frac{m^{\prime 2}}{4 r m}
$$

while the square of the Riemann tensor has the form

$$
\begin{aligned}
R_{M N R S} R^{M N R S}= & 40 \lambda^{4}+\frac{48 m^{2} e^{-4 A}}{r^{6}}+\frac{A^{\prime} m^{\prime}}{r m} \\
& \times\left(\frac{2 m^{\prime 2}}{m}-7 A^{\prime} m^{\prime}-4 m^{\prime \prime}\right)+\frac{m^{\prime 2} e^{-2 A}}{2 r^{4}} \\
& \times\left(13-\frac{9 r}{2 m}\right)+\frac{m^{\prime 2}}{r m^{2}}\left(m^{\prime \prime}-\frac{m^{\prime 2}}{4 m}+\frac{11 m^{\prime 2}}{16 r}\right) \\
& -\frac{m^{\prime \prime 2}}{r m} .
\end{aligned}
$$

From the expressions of the above quantities, ${ }^{1}$ we may see that the only singularity that makes its appearance in the scalar gravitational quantities is the black hole singularity at $r=0$. The mass function $m(y)$ is assumed to be everywhere well defined, to scale faster than $e^{2 \lambda|y|}$ and to reduce to zero away from the brane. In this way, all singular terms, both at $r=0$ and at the AdS horizon $|y| \rightarrow \infty$, vanish at a moderate distance from the brane thus restoring a regular fivedimensional spacetime.

An ansatz that manages to accommodate all the above demands and reduces to the usual mass parameter $M$ on the brane is $m(y)=M e^{-a|y|^{n}}$ where $n$ is an integer number and $a$ a positive constant. Depending on the value of $a$, the localization of the singular terms, appearing in the curvature invariant quantities, takes place at different distances from the brane but always before the AdS horizon is reached; for $a$ $\gg 2 \lambda^{2-n}$, this localization takes place at a distance smaller than the AdS radius, $\lambda^{-1}$. The above ansatz introduces no discontinuity in the mass function as long as $n>1$ since in this case $m^{\prime}(0)=0$. Discontinuities of the derivatives of the metric tensor are directly related to a localized matter tensor on the brane through the expression

$$
[K]_{\mu \nu}-h_{\mu \nu} \operatorname{Tr}[K]=-\kappa_{5}^{2} S_{\mu \nu},
$$

$K_{\mu \nu}$ being the extrinsic curvature and $h_{\mu \nu}$ the induced metric on the brane. The brackets denote the discontinuity across the brane. Taking into account the $\mathcal{Z}_{2}$ symmetry, we get

\footnotetext{
${ }^{1}$ The square of the Ricci tensor, $R_{M N} R^{M N}$, has a form similar to the one of the Riemann tensor but lacks the $1 / r^{6}$ term.
}

$$
\begin{aligned}
{[K]_{\mu \nu}=} & {\left[2 A^{\prime} h_{\mu \nu}+\delta_{\mu}^{v} \delta_{\nu}^{v} \frac{2 m^{\prime}}{r}-\frac{1}{2}\left(\delta_{\mu}^{r} \delta_{\nu}^{v}+\delta_{\mu}^{v} \delta_{\nu}^{r}\right)\right.} \\
& \left.\times \sqrt{\frac{2 m}{r}} \frac{m^{\prime}}{m}\right]_{y=0^{+}} .
\end{aligned}
$$

The first term in the above expression is the standard RS tension term proportional to $h_{\mu \nu}$ that causes the discontinuity in the second derivative of the warp factor. Nevertheless, for $m^{\prime}(y=0) \neq 0$, additional terms could also be present. This is indeed the case for $n=1$ : the discontinuity that arises can be accommodated only in the presence of additional matter strictly localized on the brane. Although this case cannot be excluded, we consider it as a rather complicated and a physically unrealistic one.

Finally we need to check the profile of the bulk energymomentum tensor components. By mere inspection of the field equations (12)-(18), we may see that, for fixed $y$, the components of the energy-momentum tensor scale as $1 / r^{k}$, where $k$ a positive number, therefore all of them go to zero at large distance from the horizon of the black hole. On the other hand, a physically sensible distribution of bulk matter demands also its localization close to the brane: a bulk matter distribution diverging at $y$ infinity would be in contradiction with the concept of localization of gravity around the brane. Figure 1 depicts the profile of the five independent "extra" components along the $y$ axis for three different values of $n$. We choose, for simplicity, $r=r_{h}=1$ and $\lambda=1$, in units of Planck mass. The case $n=1$ is qualitatively different from the one in which $n>1$ also when it comes to the distribution of bulk matter: all of the components are peaked at the location of the brane and rapidly reduce to zero as $y$ increases. On the other hand, for $n>1$, all components reach a maximum, in absolute value, at a location off the brane before reducing to zero. Small differences appear between the cases $n=2$ and $n>2$ : in the latter case, all components vanish at the position of the brane while, in the former one, some of the bulk components, including the energy-density, adopt non-zero values at the position of the brane without, however, causing any discontinuity in the Einstein tensor.

In all cases considered, the bulk matter is localized either on or off the brane while vanishing at infinity, thus creating a shell-type distribution of bulk matter. For $n>1$, the energydensity $\rho=-\hat{T}_{v}^{v}$ and radial pressure $p_{r}=\hat{T}_{r}^{r}$ satisfy a stiff equation of state, i.e. $\rho \simeq p_{r}$; however, they are not the dominant components as the off-diagonal component $\hat{T}_{r}^{v}$ prevails both at small and large distance from the brane. Almost all of the components, including the energy density, change sign at some point in the bulk, and thus one can easily show that both the weak and strong energy conditions are violated at specific regimes in the bulk. This behavior forces us to interpret them as arising from a non-ordinary distribution of matter. The importance of the non-factorized RS-Painlevé metric ansatz lies on the fact that it constitutes the first example of a spacetime with a $y$-dependent horizon that is well defined for every value of the radial coordinate $r>0$. More specifically, no divergences appear either in the components of $T_{M N}$, or in the curvature invariant quantities, at the location 

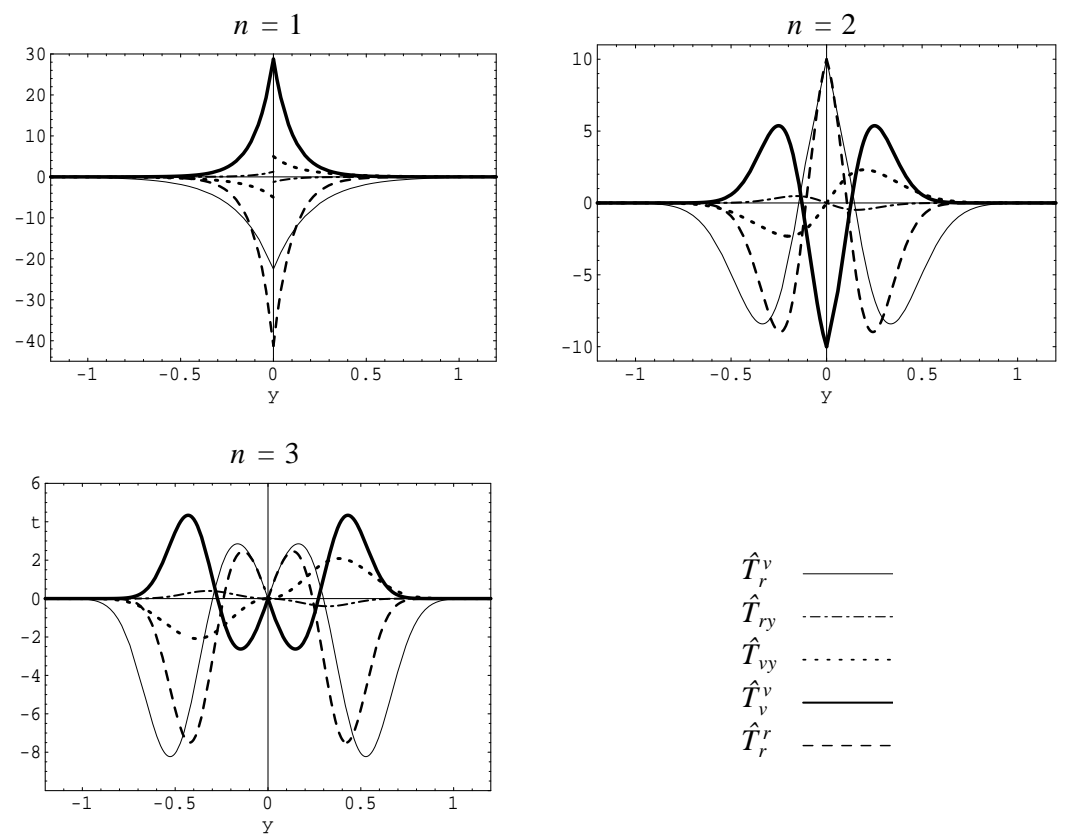

FIG. 1. The plot depicts the profile of the five independent components of the bulk energymomentum tensor along the bulk (y) coordinate for $\lambda=1, a=10$ for $n=1$, and $a=20$ for $n=2,3$ and for fixed $r=r_{h}=1$. All components create a shell-type distribution of matter in the bulk and then vanish at moderate distances from the brane. of the four-dimensional horizon of the equivalent Schwarzschild solution. The above example, as well as the one of the generalized Vaidya spacetime to be studied shortly, clearly confirm our argument that analytic black hole line elements do not generate additional bulk singularities.

\section{THE NON-FACTORIZED RS-VAIDYA SPACETIME}

We will now consider an alternative metric ansatz, that describes a Vaidya-type spacetime on the brane, of the form $[15,16]$

$$
\begin{aligned}
d s^{2}= & e^{2 A(y)}\left[-\left(1-\frac{2 m(v, y)}{r}\right) d v^{2}+2 \epsilon d v d r\right. \\
& \left.+r^{2}\left(d \theta^{2}+\sin ^{2} \theta d \varphi^{2}\right)\right]+d y^{2}
\end{aligned}
$$

where $\epsilon= \pm 1$. The $y$ dependence of the mass function $m$ is assumed to satisfy the same criteria as in the previous cases: to become trivial at the location of the brane and to cause a rapid decrease of $m$ away from it. For a $v$-independent mass function, the above line element takes the form of the Schwarzschild solution on the brane under the coordinate transformation

$$
v \rightarrow t=v-r-2 M \log (r-2 M) .
$$

Similarly to the line element presented in Eq. (10), the above metric tensor has no apparent singularity at the horizon. Therefore, we expect that no additional bulk singularities will appear. In addition, the simpler form of this particular ansatz is quite likely to lead to a simpler form for the bulk energy-momentum tensor.

In accordance with the usual Vaidya line element in four dimensions, we have kept the $v$ dependence of the mass function. For $\epsilon=+1$, the null coordinate $v$ represents the Eddington advanced time and the corresponding line element may be used to describe the collapse of a spherical shell of matter on the brane leading to the increase of its mass with time, $\partial_{v} m>0$. On the other hand, the choice $\epsilon=-1$ represents the Eddington retarded time which may be used for the study of an expanding shell of matter. Here, we will be interested in the former case, with the $v$-dependent Vaidya metric describing the spacetime on the brane during the collapse, and the $v$-independent one, or its equivalent Schwarzschild metric, describing the spacetime before or after the collapse. In what follows, unless otherwise stated, the mass function will be assumed to be both $v$ and $y$ dependent.

Let us first turn to the non-vanishing components of the five-dimensional Einstein's equations. We find that, for the metric ansatz (24), the diagonal components simply reduce to the usual ones for an AdS spacetime, namely,

$$
\begin{gathered}
6 A^{\prime 2}+3 A^{\prime \prime}=\kappa_{5}^{2}\left[-\Lambda_{B}+S_{v}^{v} \delta(y)+\hat{T}_{v}^{v}\right] \\
6 A^{\prime 2}+3 A^{\prime \prime}=\kappa_{5}^{2}\left[-\Lambda_{B}+S_{r}^{r} \delta(y)+\hat{T}_{r}^{r}\right] \\
6 A^{\prime 2}+3 A^{\prime \prime}=\kappa_{5}^{2}\left[-\Lambda_{B}+S_{\theta}^{\theta} \delta(y)+T_{\theta}^{\theta}\right] \\
6 A^{\prime 2}=\kappa_{5}^{2}\left[-\Lambda_{B}+\hat{T}_{y}^{y}\right]
\end{gathered}
$$

where $S_{\mu \nu}$ is again the stress-energy tensor localized on the wall. If we, therefore, assume that, as before, the bulk cosmological constant gives rise to the usual Randall-Sundrum form of the warp factor, the bulk energy-momentum tensor does not need to have non-trivial energy density and diagonal pressure components. Nevertheless, we must have two independent non-trivial, off-diagonal pressure components, defined by the equations 


$$
\begin{array}{r}
-\epsilon\left(\frac{4 A^{\prime} \partial_{y} m}{r}+\frac{\partial_{y}^{2} m}{r}\right)+\frac{2\left(\partial_{v} m\right) e^{-2 A}}{r^{2}}=\kappa_{5}^{2} \hat{T}_{v}^{r}, \\
e^{-2 A \frac{\partial_{y} m}{r^{2}}}=\kappa_{5}^{2} \hat{T}_{y}^{r} .
\end{array}
$$

Once again the equation for the conservation of the energymomentum tensor, $D_{M} T_{N}^{M}=0$, is trivially satisfied by the above components for an arbitrary function $m(v, y)$. The presence of the bulk components is necessary to support the non-trivial form of the mass function $m(v, y)$. Note that even for $\partial_{y} m=0$, in which case we recover the usual 4D Vaidya metric for each slice $y=$ const, the necessity of having a bulk off-diagonal pressure component, $\hat{T}_{v}^{r}$, still arises. We may therefore conclude that whenever the 4D metric has a structure that demands a non-trivial energy-momentum tensor, as for example in the case of the dynamically evolving Vaidya line element, its consistent embedding in a higherdimensional spacetime demands the introduction of a corresponding bulk energy-momentum tensor. Leaving the problem of the localization of the black-hole singularity aside, that means that the process of the production of black holes or, in general, of the collapse of matter on the brane cannot be a purely four-dimensional process that can take place in an empty bulk.

The finiteness for $r>0$ characterizes not only the components of the bulk energy-momentum tensor but also the curvature invariants derived for the line element (24), which are found to be

$$
\begin{aligned}
R= & -20 A^{\prime 2}-8 A^{\prime \prime} \\
R_{M N} R^{M N}= & 4\left(20 A^{\prime 4}+16 A^{\prime 2} A^{\prime \prime}+5 A^{\prime \prime 2}\right), \\
R_{M N R S} R^{M N R S}= & 8\left(5 A^{\prime 4}+4 A^{\prime 2} A^{\prime \prime}+2 A^{\prime \prime 2}\right. \\
& \left.+6 \frac{m^{2}(v, y)}{r^{6}} e^{-4 A(y)}\right),
\end{aligned}
$$

for an arbitrary warp factor function $A(y)$. Similarly to the case studied in the previous section, the above scalar quantities do not have any new singularities in the bulk. Formally they are identical to the ones derived for a RS-Schwarzschild line element [although in that case $m(v, y)=m=$ const]; however, in this case, the scaling of the horizon with the distance from the brane can lead to the localization of the black hole singularity.

In the following, let us consider $A(y)=-\lambda|y|$ and the simple ansatz $m(v, y)=m(v) e^{-a|y|^{n}}$, where $n$ is an integer number and $a$ a positive constant. For large enough values of $a$, the horizon reduces to zero much faster than the square of the warp factor so that the last, singular term in the expression of the square of the Riemann tensor goes to zero as $y$ $\rightarrow \infty$ unlike the black string solution (again, for $a \gg 2 \lambda^{2-n}$, the localization takes place at a distance smaller than the AdS radius $\lambda^{-1}$ ). As in the case of the Painlevé metric, the simplest choice would be to consider a mass function continuous in $y$. In the most general case of discontinuous $m$, and assuming $\mathcal{Z}_{2}$ symmetry, we have for the extrinsic curvature

$$
K_{\mu \nu}=-\Gamma_{\mu \nu}^{y}=A^{\prime} h_{\mu \nu}+\left.\delta_{\mu}^{v} \delta_{\nu}^{v} \frac{\partial_{y} m(v, y)}{r}\right|_{y=0},
$$

and for the localized matter tensor

$$
\kappa_{5}^{2} S_{\mu \nu}=\left[6 A^{\prime} h_{\mu \nu}-2 \delta_{\mu}^{v} \delta_{\nu}^{v} \frac{\partial_{y} m(v, y)}{r}\right]_{y=0^{+}} .
$$

For the RS decaying exponential warp factor, we get the usual tension term plus an additional term for the $S_{v v}$ component provided $\partial_{y} m(v, y=0) \neq 0$. For $n>1$, this quantity is zero as before, and the brane is characterized only by a constant tension. For $n=1$, however, the mass function is discontinuous on the brane and the field equations demand the introduction of a null fluid on the brane characterized by a single non-vanishing component $S_{v v}$. We consider this case as a non-realistic one; however, we include it in the following discussion as a choice consistent with the field equations.

Both bulk components scale as $1 / r$ at large distances from the horizon, taken to be $r_{h}=2 m=1$ at a fixed time $v$ $=$ const, and thus vanish asymptotically. In order to study the profile of the components of the bulk energy-momentum tensor along the extra dimension, we may set $r=r_{h}$ and $\lambda=1$, again in units of Planck mass. Since we study the collapse of brane matter, we further assume that $\epsilon=+1$ and $\partial_{v} m>0$. We have denoted as $\hat{T}_{v}^{r(s)}$ the "static part" of $\hat{T}_{v}^{r}$, i.e. the first two terms in Eq. (30), that represent the value of this component in the two static limits, either before or after the collapse of the brane shell. On the other hand, $\hat{T}_{v}^{r(a)}$ stands for the total expression containing also the non-static term proportional to $\partial_{v} m$, and gives the value of the component $d u r-$ ing the collapsing phase. The profile of the off-diagonal components of the energy-momentum tensor, $\hat{T}_{v}^{r}$ and $\hat{T}_{y}^{r}$, along the $y$ axis, is depicted in Fig. 2. As desired, both components, either static or non-static, are "localized" around the brane and vanish exponentially fast as $y \rightarrow \infty$.

We can see, once again, that the bulk matter distribution is qualitatively different depending on the value of $n$. For $n$ $=1$, we can see that some bulk components have a discontinuity at the brane position while, for $n>1$, all of them are continuous across the brane. Focusing on the more realistic case of $n>1$, we note that before or after the collapse, when the line element is taken to be static, the $\hat{T}_{v}^{r}$ off-diagonal pressure component reaches a negative minimum value at some distance off the brane before going to zero at larger distances. As the collapse begins and $\partial_{v} m$ starts to increase, the $\hat{T}_{v}^{r}$ component increases too, reaching a maximum positive value off the brane. As the collapse reaches its end, the $\hat{T}_{v}^{r}$ component falls back into its static, negative value $\hat{T}_{v}^{r(s)}$. We may thus conclude that while a bulk, negative $\hat{T}_{v}^{r}$ pressure component is necessary to support a $y$-decaying horizon, a symmetric positive pressure barrier is necessary in the bulk during dynamical collapse on the brane, when we have a 


$$
n=1
$$
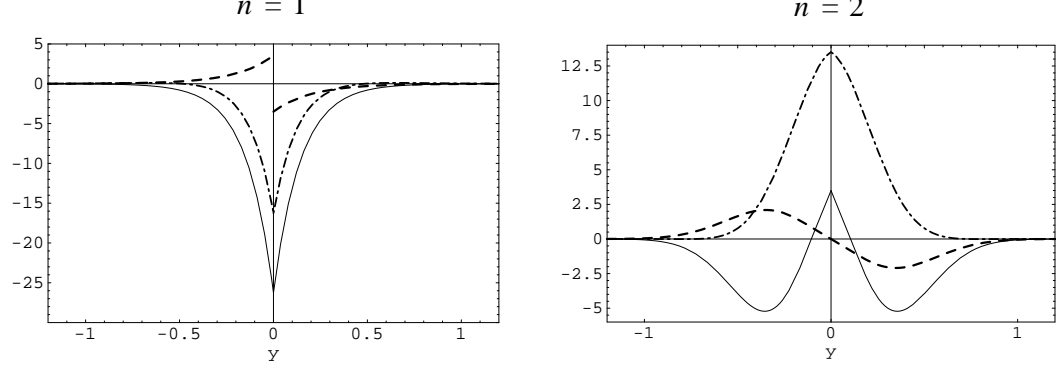

$n=3$

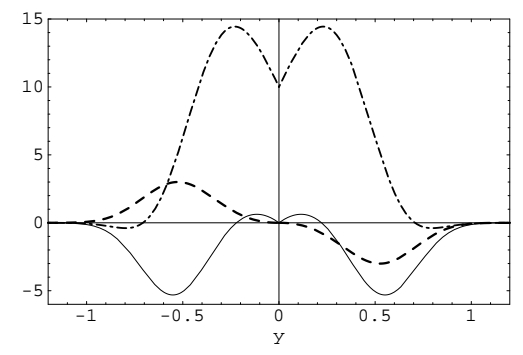

non-trivial in-flow of energy. The remaining off-diagonal component $\hat{T}_{y}^{r}$ is not affected by the process of collapse on the brane.

The above bulk energy-momentum tensor has zero energy-density and diagonal pressure components, so its interpretation by means of an ordinary matter distribution (a single scalar or gauge field) is not possible. Specific attempts towards this direction have proved to be unsuccessful similarly to the analysis performed in [11]. However, the demand for the existence of only pressure bulk components, which are not accompanied by an energy density, is not unusual in brane models. Such components have been introduced in single [17] or two brane [18] models in order to modify the topology of the extra dimension. In all those examples, the bulk pressure component was merely a reflection of what existed or was taking place on the brane. The present situation is very similar to the aforementioned one as the introduced pressure components keep the topological structure of a brane black hole embedded in a higher-dimensional spacetime close to the brane. In [17], it was shown that the existence of pressure components without a corresponding energy-density in the bulk cannot be attributed to the presence of an ordinary scalar field with a standard kinetic and potential term. An interpretation of such a bulk energymomentum tensor was finally given in terms of a scalar field with a non-linear kinetic term of the form $\left(\partial_{M} \phi \partial^{M} \phi\right.$ $\left.-c^{2}\right)^{2}$. By considering similar models in the present case, as well as models with two scalar fields and bulk Lagrangian of the form ${ }^{2} \mathcal{L}_{B} \sim \partial_{M} \phi \partial^{M} \chi$, we were indeed able to construct a bulk energy-momentum tensor with the desired nonvanishing components. Although these models could also accommodate a $y$-dependent black hole horizon, none of the models constructed could support a decaying horizon necessary for the localization of the black hole singularity. In prin-

\footnotetext{
${ }^{2}$ In all cases, the potential $V$, either self-interacting or crossinteracting, had to be trivially zero.
}

ciple, however, a model of scalar fields with a structure $\partial_{M} \phi_{a} \partial^{M} \phi_{b} K_{a b}(\phi, \partial \phi)$ could lead to such a bulk energymomentum tensor.

\section{CONCLUSIONS}

The construction of black hole solutions in the context of warped brane models has proven to be surprisingly difficult due to the difficulty in localizing the black hole singularity near the brane. Although some progress has been made in the numerical construction of such solutions [7], a satisfactory analytical solution of this form, which would give us valuable information regarding the nature of the gravitational collapse on a brane embedded in a higher-dimensional spacetime, is still missing.

In Ref. [11] an attempt was made to localize the black hole singularity near the brane and, at the same time, avoid the singularity at the AdS horizon by introducing a dependence of the 4D horizon on the coordinate along the extra dimension. An exponentially "decaying" form of the black hole horizon in terms of the $y$ coordinate was shown to achieve both of the aforementioned tasks. Nevertheless, this non-factorizable line element had to be sustained by a bulk energy-momentum tensor, which although localized around the brane, was of an exotic nature and was plagued by an additional singularity located at the 4D horizon, a singularity that was also present in the expressions of the curvature invariant quantities.

In this paper we have pursued further the quest for localized black holes by providing answers to questions that remained open after the end of our previous work, such as the following: Is the additional singularity a generic feature of every 5D spacetime with a non-factorized line element? Can alternative line elements be found that are characterized by a similar scaling of the black hole horizon and that do not possess this problem? Does the corresponding bulk energymomentum tensor have to be always of an exotic nature?

We have started our analysis by demonstrating that non- 
factorized line elements which are not analytic at the horizon are always accompanied by the appearance of singular terms in the expressions of the higher-dimensional curvature invariant quantities. This leads to the manifestation of the 4D black-hole horizon as a true spacetime singularity in the higher-dimensional spacetime. The above result paved the way for the introduction on the brane of black hole line elements related by coordinate transformations to the Schwarzschild one, thus describing the same 4D spacetime, that were analytic at the horizon. We considered two ansätze of this form, the Painlevé and Vaidya line elements, which were then embedded in a non-factorized way in a 5D warped brane model. In both cases, the resulting 5D spacetime was shown to be well defined for every $r>0$ while the scaling of the horizon with the extra coordinate led to the localization of the black hole singularity, at $r=0$. In the case of the Painlevé line element this scaling demands the introduction of a regular, shell-type but highly complicated bulk energymomentum tensor that clearly cannot be attributed to a standard form of matter. On the other hand, the Vaidya line element proves to be a much more attractive, natural choice. Despite the scaling of the horizon, the 5D spacetime has the same topological structure as the one of a factorized RSSchwarzschild spacetime. This simplicity is also reflected in the form of the bulk energy-momentum tensor: only two shell-like off-diagonal pressure components must exist in the bulk while the energy density and diagonal pressure components are zero. Motivated by previously studied brane models with similar form of bulk matter, we speculate that this particular bulk energy-momentum tensor may be attributed not necessarily to an exotic form of matter but simply to a non-ordinary one, i.e. scalar field theories with non-linear kinetic terms.

\section{ACKNOWLEDGMENT}

I.O. and K.T. acknowledge the financial support of the EU RTN contract No. HPRN-CT-2000-00152. K.T. acknowledges also the support of the EU RTN with contract No. HPRN-CT-2000-00148. He also wishes to thank the CERN Theory Division for its hospitality.

\section{APPENDIX}

For the $D$-dimensional spacetime described by the metric tensor given in Eq. (6), we obtain the following nonvanishing components of the Christoffel symbols:

$$
\begin{gathered}
\Gamma_{\rho \sigma}^{\mu}=\hat{\Gamma}_{\rho \sigma}^{\mu}, \quad \Gamma_{\rho s}^{\mu}=\delta_{\rho}^{\mu} \partial_{s} A+\frac{1}{2} \hat{g}^{\mu \nu} \hat{g}_{\rho \nu, s}, \\
\Gamma_{r s}^{m}=\widetilde{\Gamma}_{r s}^{m}, \quad \Gamma_{\rho \sigma}^{m}=-\gamma^{m n} e^{2 A}\left(\partial_{n} A \hat{g}_{\rho \sigma}+\frac{1}{2} \hat{g}_{\rho \sigma, n}\right),
\end{gathered}
$$

where $\hat{\Gamma}_{\rho \sigma}^{\mu}$ and $\widetilde{\Gamma}_{r s}^{m}$ denote the components of the Christoffel symbols being evaluated exclusively in terms of the $\hat{g}_{\mu \nu}$ and $\gamma_{m n}$ metric tensor, respectively. By using the above quantities, the relevant components of the $D$-dimensional Ricci tensor are written as

$$
\begin{aligned}
R_{\mu \nu}= & \hat{R}_{\mu \nu}-\frac{e^{2 A}}{2 \sqrt{-g}} \partial_{m}\left[\sqrt{-g} \gamma^{m n} \hat{g}_{\mu \nu, n}\right] \\
& +\frac{1}{2} e^{2 A} \hat{g}^{\rho \lambda} \gamma^{m n} \hat{g}_{\mu \rho, m} \hat{g}_{\nu \lambda, n}-e^{2 A} \hat{g}_{\mu \nu}\left[(p+1) \partial^{m} A \partial_{m} A\right. \\
& \left.+\widetilde{D}^{m} \widetilde{D}_{m} A+\partial^{m} A \partial_{m} \ln \sqrt{-\hat{g}}\right]-\frac{p+1}{2} e^{2 A} \partial^{m} A \hat{g}_{\mu \nu, m} \\
R_{m n}= & \widetilde{R}_{m n}-\widetilde{D}_{m} \widetilde{D}_{n} \ln \sqrt{-\hat{g}}-\left[\partial_{m} A \partial_{n} \ln \sqrt{-\hat{g}}\right. \\
& \left.+\partial_{n} A \partial_{m} \ln \sqrt{-\hat{g}}\right]-(p+1)\left[\widetilde{D}_{m} \widetilde{D}_{n} A+\partial_{m} A \partial_{n} A\right] \\
& -\frac{1}{4} \hat{g}^{\alpha \beta} \hat{g}^{\rho \lambda} \hat{g}_{\alpha \lambda, n} \hat{g}_{\rho \beta, m},
\end{aligned}
$$

where $\sqrt{-g}$ and $\sqrt{-\hat{g}}$ denote the determinant of the $D$-dimensional and $(p+1)$-dimensional spacetime, respectively, and $\widetilde{D}_{m}$ the covariant derivative with respect to the "extra" spacetime.

Contracting $R_{M N}$ by $g^{M N}$, we may determine the expression of the higher-dimensional Ricci scalar which is found to be

$$
\begin{aligned}
R= & e^{-2 A} \hat{R}+\widetilde{R}-2 \widetilde{D}^{m} \widetilde{D}_{m} \ln \sqrt{-\hat{g}}-\partial^{m} \ln \sqrt{-\hat{g}} \partial_{m} \ln \sqrt{-\hat{g}} \\
& +\frac{1}{4} \hat{g}^{\mu \nu} \hat{g}^{\rho \lambda} \hat{g}_{\nu \lambda, m} \hat{g}_{\mu \rho}{ }^{, m}+\frac{1}{2} \hat{g}_{\mu \nu, m} \hat{g}^{\mu \nu, m}-(p+2) \partial^{m} A \\
& \times\left[(p+1) \partial_{m} A+2 \partial_{m} \ln \sqrt{-\hat{g}}\right]-2(p+1) \widetilde{D}^{m} \widetilde{D}_{m} A .
\end{aligned}
$$

[1] I. Antoniadis, Phys. Lett. B 246, 377 (1990); N. ArkaniHamed, S. Dimopoulos, and G.R. Dvali, ibid. 429, 263 (1998); Phys. Rev. D 59, 086004 (1999); I. Antoniadis, N. ArkaniHamed, S. Dimopoulos, and G.R. Dvali, Phys. Lett. B 436, 257 (1998).

[2] L. Randall and R. Sundrum, Phys. Rev. Lett. 83, 3370 (1999).

[3] L. Randall and R. Sundrum, Phys. Rev. Lett. 83, 4690 (1999).

[4] J. Lykken and L.J. Randall, J. High Energy Phys. 06, 014 (2000); K. Skenderis and P.K. Townsend, Phys. Lett. B 468, 46
(1999); K. Behrndt and M. Cvetic, ibid. 475, 253 (2000); A. Chamblin and G.W. Gibbons, Phys. Rev. Lett. 84, 1090 (2000); O. DeWolfe, D.Z. Freedman, S.S. Gubser, and A. Karch, Phys. Rev. D 62, 046008 (2000); C. Csaki, J. Erlich, T.J. Hollowood, and Y. Shirman, Nucl. Phys. B581, 309 (2000); S.B. Giddings, E. Katz, and L.J. Randall, J. High Energy Phys. 03, 023 (2000); H. Kudoh and T. Tanaka, Phys. Rev. D 64, 084022 (2001); 67, 044011 (2003).

[5] R. Emparan, G.T. Horowitz, and R.C. Myers, J. High Energy 
Phys. 01, 007 (2000); J. Garriga and M. Sasaki, Phys. Rev. D 62, 043523 (2000); A. Chamblin, C. Csaki, J. Erlich, and T.J. Hollowood, ibid. 62, 044012 (2000); N. Dadhich, R. Maartens, P. Papadopoulos, and V. Rezania, Phys. Lett. B 487, 1 (2000); S. Nojiri, O. Obregon, S.D. Odintsov, and S. Ogushi, Phys. Rev. D 62, 064017 (2000); A. Chamblin, H.S. Reall, H.a. Shinkai, and T. Shiromizu, ibid. 63, 064015 (2001); I. Giannakis and H.c. Ren, ibid. 63, 024001 (2001); 64, 065015 (2001); Phys. Lett. B 528, 133 (2002); M.S. Modgil, S. Panda, and G. Sengupta, Mod. Phys. Lett. A 17, 1479 (2002).

[6] M. Bruni, C. Germani, and R. Maartens, Phys. Rev. Lett. 87, 231302 (2001); M. Govender and N. Dadhich, Phys. Lett. B 538, 233 (2002); M. Rogatko, Phys. Rev. D 64, 064014 (2001); R. Emparan and H.S. Reall, Phys. Rev. Lett. 88, 101101 (2002); T. Wiseman, Phys. Rev. D 65, 124007 (2002); R. Casadio, A. Fabbri, and L. Mazzacurati, ibid. 65, 084040 (2002); S.I. Vacaru and D. Singleton, Class. Quantum Grav. 19, 3583 (2002); R. Casadio and L. Mazzacurati, Mod. Phys. Lett. A 18, 651 (2003); R. Emparan, A. Fabbri, and N. Kaloper, J. High Energy Phys. 08, 043 (2002); P. Kanti, I. Olasagasti, and K. Tamvakis, Phys. Rev. D 66, 104026 (2002); G. Kofinas, E. Papantonopoulos, and V. Zamarias, ibid. 66, 104028 (2002); S. Shankaranarayanan and N. Dadhich, gr-qc/0306111; K.A. Bronnikov, V.N. Melnikov, and H. Dehnen, Phys. Rev. D 68 , 024025 (2003).

[7] T. Wiseman, Class. Quantum Grav. 20, 1137 (2003); H. Kudoh, T. Tanaka, and T. Nakamura, Phys. Rev. D 68, 024035 (2003).

[8] P.C. Argyres, S. Dimopoulos, and J. March-Russell, Phys. Lett. B 441, 96 (1998); S.B. Giddings and S. Thomas, Phys. Rev. D 65, 056010 (2002); S. Dimopoulos and G. Landsberg, Phys. Rev. Lett. 87, 161602 (2001); R. Emparan, G.T. Horowitz, and R.C. Myers, ibid. 85, 499 (2000); M.B. Voloshin, Phys. Lett. B 518, 137 (2001); D.M. Eardley and S.B. Giddings, Phys. Rev.
D 66, 044011 (2002); R. Casadio and B. Harms, Int. J. Mod. Phys. A 17, 4635 (2002); K. Cheung, Phys. Rev. Lett. 88, 221602 (2002); E.J. Ahn, M. Cavaglia, and A.V. Olinto, Phys. Lett. B 551, 1 (2003); V. Cardoso and J.P. Lemos, ibid. 538, 1 (2002); A. Chamblin and G.C. Nayak, Phys. Rev. D 66, 091901(R) (2002); V. Frolov and D. Stojkovic, Phys. Rev. Lett. 89, 151302 (2002); P. Kanti and J. March-Russell, Phys. Rev. D 66, 024023 (2002); 67, 104019 (2003); Y. Uehara, hep-ph/0205068; A.V. Kotwal and C. Hays, Phys. Rev. D 66, 116005 (2002); T. Han, G.D. Kribs, and B. McElrath, Phys. Rev. Lett. 90, 031601 (2003); D. Ida, K.y. Oda, and S.C. Park, Phys. Rev. D 67, 064025 (2003); I. Mocioiu, Y. Nara, and I. Sarcevic, Phys. Lett. B 557, 87 (2003); A. Chamblin, F. Cooper, and G.C. Nayak, hep-ph/0301239.

[9] A. Chamblin, S.W. Hawking, and H.S. Reall, Phys. Rev. D 61, 065007 (2000).

[10] R. Gregory and R. Laflamme, Phys. Rev. Lett. 70, 2837 (1993).

[11] P. Kanti and K. Tamvakis, Phys. Rev. D 65, 084010 (2002).

[12] N. Kaloper, Phys. Rev. D 60, 123506 (1999); H.B. Kim and H.D. Kim, ibid. 61, 064003 (2000); A. Karch and L. Randall, J. High Energy Phys. 05, 008 (2001); T. Hirayama and G. Kang, Phys. Rev. D 64, 064010 (2001).

[13] P. Painlevé, Acad. Sci., Paris, C. R. 173, 677 (1921).

[14] P. Kraus and F. Wilczek, Mod. Phys. Lett. A 9, 3713 (1994); M.K. Parikh and F. Wilczek, Phys. Rev. Lett. 85, 5042 (2000).

[15] P.C. Vaidya, Nature (London) 171, 260 (1953).

[16] E. Poisson and W. Israel, Phys. Rev. D 41, 1796 (1990); C. Barrabes and W. Israel, ibid. 43, 1129 (1991).

[17] P. Kanti, I.I. Kogan, K.A. Olive, and M. Pospelov, Phys. Lett. B 468, 31 (1999); Phys. Rev. D 61, 106004 (2000).

[18] C. Csaki, M. Graesser, L. Randall, and J. Terning, Phys. Rev. D 62, 045015 (2000); P. Kanti, K.A. Olive, and M. Pospelov, Phys. Lett. B 481, 386 (2000); Phys. Rev. D 62, 126004 (2000). 